\title{
Stiff Neck Associates with Life Styles in Multiphasic Health Testing and Service
}

\author{
Toshiyuki Shibosawa ${ }^{1}$; Hiroki Sugimori ${ }^{2}$;oshio Ogino ${ }^{1}$; Shigeyuki Takeuchi ${ }^{1}$; Makoto Yuki ${ }^{1}$; and Asami Kishida ${ }^{1}$ \\ ${ }^{1}$ Toma Hospital MHTS Center, and ${ }^{2}$ Department of Preventive Medicine, St. Marianna University School of Medicine.
}

\begin{abstract}
We conducted a survey of 11,734 health-checkups participants (7,774 male and 3,960 female) regarding actual condition of 'stiff neck' that was one of the major complaints reported in The Basic Research of National Living in the 1998 fiscal year, by The Japanese Ministry of Health and Welfare. The number of people who suffered from stiff neck was as follows: 2,218 male (28.5\% of all male participants); 1,979 female (50.0\% of all female participants). Prevalence of stiff neck was significantly higher in female than that in males, as the same as in the other previous reports $(p<0.05$, chi-square). Stiff neck also significantly related with lumbago. Furthermore, those who suffered from stiff neck had the tendency of the following life styles: inactive in daily life, less exercise, feel more stress, not drink, and not smoke. We would like to make use of the results of this research for the consultation to improve life styles.
\end{abstract}

Key Words Stiff Neck, Health-Checkups, Life Style, Epidemiology, Descriptive Study

\section{INTRODUCTION}

According to the Basic Research of National Living published in the 1998 fiscal year by the Japanese Ministry of Health and Welfare, 'stiff neck' was the most common complaint among adult women, where lumbago that was the most common complaint and followed by 'stiff neck' among adult men. Although the definition of the stiff neck is obscure, it is regarded as the pain or stiff indisposition on the nuchal and trapezius muscle or the scapular region, sometimes involving the indurations of the muscle that is often eased by simple massaging.

Recently, laborsaving and labor related automation has been developed in the workplace. The home environment has also changed rapidly, taking this into account people are obliged to live continually without the sufficient contraction of muscles, making the muscles stiff and weak, causing an increase in stress of the nuchal and trapezius muscles which causes stiff neck. In most of these cases, no pathological problem is found on the affected part, and "stiff neck" is regarded as the occupational disorder of the neck, shoulder and arms defined by the committee of neck, shoulder, arms syndrome (KEI-KEN-WAN-SHOKOGUN) of the Japan Society for Occupational Health.

When physical conditions cause stiff neck, there are several reasons: neck spondylosis, neck discopathy, neck disk herniation, abnormal labile of the neck caused by bull neck or square shoulders, ${ }^{1)}$ sloping shoulders, diseases caused by the cervical vertebra or brachial nerve such as the thoracic outlet syndrome, loose

\footnotetext{
Received: January 15, 2002.

1 Address; Toma Hospital MHTS Center, Suhehiro 2-137, Kumagaya, Saitama, 360-0031 Japan. TEL; 81-48-522-0600 (Ext. 149), FAX; 81-48-525-8692

${ }^{2}$ Address; Department of Preventive Medicine, St. Marianna University School of Medicine, Sugao 2-16-1, Miyamae-ku, Kawasaki, Kanagawa, 216-8555 Japan
}

shoulder, arthrosteitis for instance caused by bad posture, menopausal disorder, muscle contraction headache, psychosomatic disorder, autonomic nerve imbalance, high blood pressure, arteriosclerosis, the circulatory disease such as myocardial infarction, angina pectoris, digestive disease such as the gallbladder, liver, stomach disorder, diseases caused by dental, ophthalmic, otological reasons.

Therefore, the purpose of this descriptive study is to demonstrate the actual condition of stiff neck by conducting a survey on people who had health-checkups, and analyzing the relationship between the results of the health check and the questions they had answered.

\section{SUBJECTS AND METHODS}

The total of 11,734 (7,774 male and 3,960 female) eligible health-checkups subjects, ages ranging from teens to eighties, were registered from the participants of Toma Hospital Multiphasic Health Testing and Service (MHTS) Center during the period between January and October 1999. The interview (anamneses) was also made for subjects regarding the actual condition of several items: stiff neck, lumbago, stress, daily activity, physical exercise, drinking, and smoking. Furthermore, we examined eyesight, eye pressure, and hearing. We investigated the relationship between the results of these items and stiff neck.

A level of $p<0.05$ was accepted as statistically significance. Statistical analysis including chi-square test was performed by using Microsoft Excel (R) 2000 programs (Microsoft Corporation). Odds ratio (OR) was calculated and then Woolf's method was used to obtain its $95 \%$ confidence interval $(95 \% \mathrm{CI})$. Our study was conducted in accordance with the recommendations outlined in the Declaration of Helsinki (revised in 1983, 2000), and with utmost caution to subjects' personal confidentiality.

\section{RESULTS}

The number subjects who have suffered from stiff neck was 2,218 in men, which is $28.8 \%$ of the total number of male participants, and 1,979 in women, which is $50.0 \%$ of the total number of female participants. Prevalence of stiff neck was significantly larger in female than that in males ( $p<0.05$, chi-square). Examining the result of female stiff neck sufferers by age, at the age 20 to 69 , it was approximately $50 \%$ regardless of age; at the age 70 to 79 it decreased to $36.7 \%$. In the case of males, the highest number were in their twenties at $35.3 \%$, the figures stabilized to $28-30 \%$ at ages 30 to 59 , and reduced to $20.3-22.5 \%$ at the age $60-79$.

Stiff neck sufferers did not necessarily increase as age increases, however there was a tendency where the number of sufferers did decrease at the ages of 60 or older in males, 70 or older in the females (Fig. 1). Moreover, the number of stiff neck sufferers was 


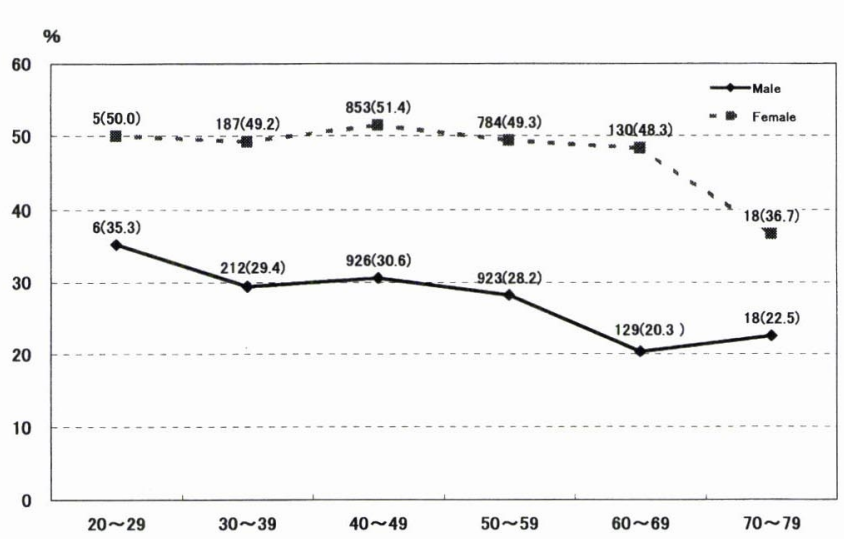

Fig. 1 Prevalence of stiff neck by age-sex groups.

2.9 times as many as that of lumbago sufferers in females, and twice that in males. Five hundreds and thirteen out of 2.218 male stiff neck sufferers had lumbago at the same time, which was $23.1 \%$, and this prevalence was significantly higher than that of non-stiff neck male sufferers, which was $10.9 \%$ (OR 2.46, 95\% CI 2.16-2.80). In the same way, $22.9 \%$ of 1,979 female stiff neck sufferers had lumbago; this ratio was significantly higher than that of the lumbago sufferers among non-stiff neck female sufferers (OR2.24, 95\% CI 1.88-2.66).

The results regarding stress-related stiff neck were as follows: $76.2 \%$ of the male stiff neck sufferers felt stress, $57.8 \%$ of the males who had no stiff neck felt stress, $76.7 \%$ of the female stiff neck sufferers felt stress, $60.7 \%$ of the females who had no stiff neck felt stress. On the other hand, $23.0 \%$ of the male stiff neck sufferers did not feel stress, $40.6 \%$ of the males who had no stiff neck did not feel stress; $21.9 \%$ of the female stiff neck sufferers did not feel stress, $37.6 \%$ of the females who had no stiff neck did not feel stress. From these results, we found that the both male and female stiff neck sufferers tend to feel more stress than the people without stiff neck.

Neck ache (on the nuchal and trapezius muscle) was seen in $3.4 \%$ males of the total number of male participants, and in $4.8 \%$ females, in $3.9 \%$ of all. Neck ache was seen in $67.6 \%$ of male stiff neck sufferers, and in $80.5 \%$ females, in $73 \%$ of all. These ratios were the higher than that of stiff neck sufferers among the total number of all participants, which was $28.5 \%$ in the males, $50.0 \%$ in the females, $35.5 \%$ in all.

The ratios of the people who experienced the whiplash among the total number of all participants were $3.4 \%$ in the males, $4.0 \%$ in the females, $3.6 \%$ in all. The ratios of stiff neck sufferers among them were $70.0 \%$ in the males, $49.4 \%$ in the females, $57.1 \%$ in all; and these ratios were higher than that of the stiff neck sufferers among the total number of all participants.

Of those who experienced disorder of the neck, shoulder, or arms, were $0.5 \%$ in the males, $1.4 \%$ in the females, and $0.8 \%$ in all among the total number of all participants. Although these ratios were below that of neck ache sufferers or persons who had experienced whiplash, the ratio of stiff neck sufferers among them were $65.9 \%$ in the males, $81.8 \%$ in the females, $75.0 \%$ in all; these ratios were higher than the ones of stiff neck sufferers among the total number of all participants. Also these ratios were higher than that of stiff neck sufferers among the people who had experienced whiplash or suffer with neck ache.

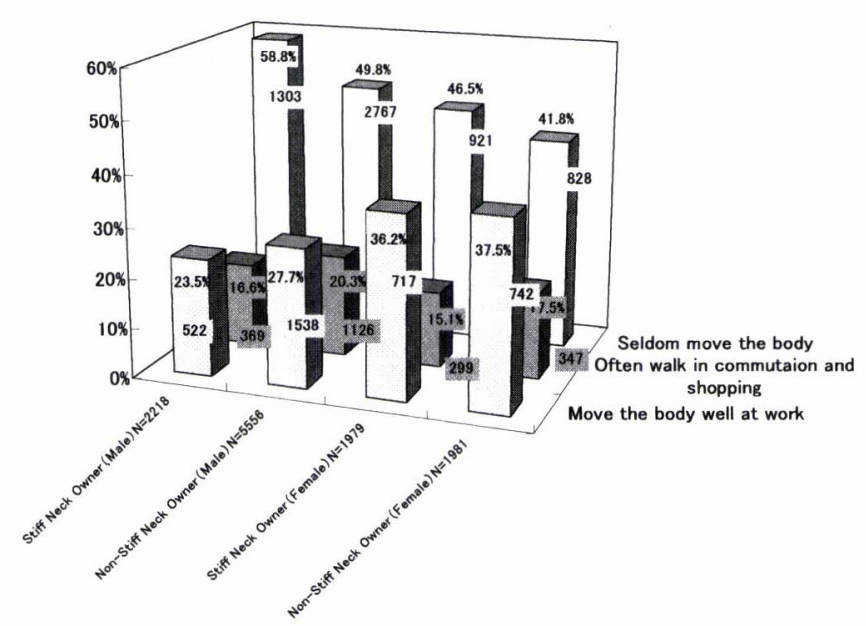

Fig. 2 Stiff neck and daily activity by sex.

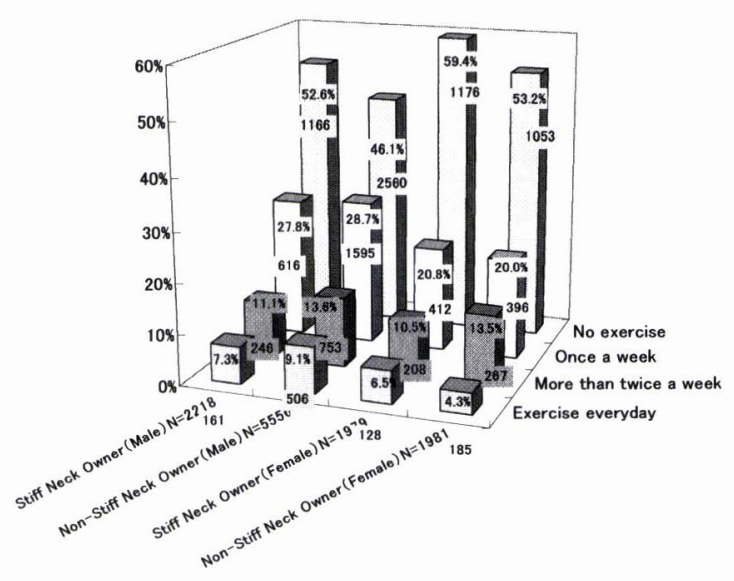

Fig. 3 Stiff neck and physical exercise by sex.

The relationship between stiff neck and daily activity was investigated. Among the subjects who seldom move their body, there were significantly more stiff neck sufferers $(58.8 \%$ in the males, $46.5 \%$ in the females) than with the persons without stiff neck ( $49.8 \%$ in the males, $41.8 \%$ in the females). Also, among persons who often travel to work and go shopping on foot, there were fewer stiff neck sufferers $(16.6 \%$ in the males, $15.1 \%$ in the females) than with persons without stiff neck $(20.3 \%$ in the males, $17.5 \%$ in the females). Moreover, among persons whose work required some daily activity demand, there were less stiff neck sufferers $(23.5 \%$ in males, $36.2 \%$ in the females) than that of persons without stiff neck $(27.7 \%$ in the males $37.5 \%$ in females) (Fig. 2). Thus, persons who were more active in a daily life tended to have less stiff neck both for males and females.

The relationship between those who have the opportunity to do physical exercise and those without stiff neck was investigated. The result is as below (Fig. 3). Frequency of males who exercise every day was $7.3 \%$ among stiff neck sufferers, whereas $9.1 \%$ among the persons without stiff neck. Frequency of males who exercise more than twice a week was $11.1 \%$ among stiff neck sufferers, whereas $13.6 \%$ among the persons without stiff neck. Males who exercise about once a week was $27.8 \%$ among stiff neck sufferers, whereas $28.7 \%$ among the persons without stiff neck. Frequency of males who didn't exercise was $52.6 \%$ among 
stiff neck sufferers, whereas $46.1 \%$ among the persons without stiff neck. We observed that there seemed to be less stiff neck sufferers among persons who had more opportunity to exercise; the result of female participants was the same as the males.

The relation between stiff neck and consuming alcoholic beverages was also investigated. Among the persons who didn't drink, there were $30.5 \%$ male, $49.5 \%$ female stiff neck sufferers and total $42.3 \%$. And among the persons, who drank, there were $28.1 \%$ male, $51.3 \%$ female stiff neck sufferers, and total $31.7 \%$. Among the persons who didn't drink, there were more stiff neck sufferers, and the ratios were higher than that of stiff neck sufferers among the total number of male and all participants. We could observe that persons who didn't drink might have more stiff neck.

Moreover, the relation between stiff neck and smoking was investigated. Among the non-smoking persons, there were $35.1 \%$ stiff neck sufferers, $31.5 \%$ among the persons who stopped smoking, $28.6 \%$ among the persons who reduced quantity of smoking, and $31.9 \%$ among the persons who smoked. Among the Smokinggroup (the persons who smoked or reduced quantity of smoking), the ratios were less than the ratio of stiff neck sufferers among the total number of all participants $(35.8 \%)$. Stiff neck sufferers among the Non-smoking group (the persons who didn't smoke or stopped smoking) was $34 \%$ which was larger than those among the Smoking-group (31.5\%), though the ratio among the persons who stopped smoking was few $(31.5 \%)$. We could observe that persons who smoke had less stiff neck.

We could not find any significant relationships between the stiff neck group and the non-stiff neck group, regarding eyesight, eye pressure, eyeground findings and hearing.

\section{DISCUSSION}

While we were researching about stiff neck by means of anamneses to the participants who had taken health-checkups, the problem was that the people had different definition about the condition of stiff neck. In our Toma hospital MHTS Center, relevant to stiff neck was asked in three different the questionnaire categories of MHTS and the ratio of stiff neck sufferers were as follows, stiff neck related to high blood pressure $(35.8 \%)$, related to disease of bone, joint and muscle $(33.1 \%)$, and related to disease of eye and brain $(17.8 \%)$. We had examined the result from high blood pressure where stiff neck sufferers were the highest. While researching the actual condition of stiff neck by inquiring, it's important to note that the their conceptual definition of stiff neck were influenced and different for each person depending on the related question items.

According to the Basic Research of the National Living by the Japanese Ministry of Health and Welfare in 1998, the number of the female stiff neck sufferers became larger than ever to 122.8 (per 1,000 of population). This number is 1.6 times as large as the one of 12 years ago, which was 77.8 (per 1,000 of population) in 1986. As for the number of the male stiff neck sufferers, it became 1.7 times larger than the one of 12 years ago from 34.2 (per 1,000 of population) to 57.2 (per 1,000 of population). Both male and female stiff neck sufferers have increased in the last 12 years. This might be due to the rapid development of a laborsaving society, which enabled people to do a job with limited movement; the four limb truncal muscles were therefore not utilized causing muscle weakness and an increase in mental stress.
If we look at the distribution by age of these statistics we can see that ages 15-64 has the highest incident rate, next is the 65-74 age group, where ages $75-84$ are 7 th and the over $85 \mathrm{~s}$ are 12 th respectively. Although according to statistics, the incident rate of lumbago sufferers, joint aches and pain in the arms and legs, clouded vision, impaired hearing and forgetfulness all increase with age, the incident rate of stiff neck sufferers decrease. ${ }^{2)}$

According to results, among the 34,781 male, 44,660 female, total 79,441 people aged over 20 , there were more stiff neck sufferers in females $(62.3 \%)$ than in males $(42.2 \%)$. As for distribution by age of these statistics, both males and females in their forties were of the highest incident rate, where men and women in their seventies the lowest. ${ }^{3)}$ The ratios of stiff neck sufferers among the people who had the health-checkups at Toma Hospital MHTS Center, $28.5 \%$ were males, $50.0 \%$ females and the result was the same as the other reports at the point of there being more in the females than in the males.

One of the possible reasons why there were more stiff neck sufferers in the females might be that the women's muscle progress less than men's and the female hormone environment causes loose ligaments and an unstable shoulder joint. ${ }^{4)}$

Stiff neck, lumbago are conditions related to the life style which arises out of the individual's living habit and working style as is with cancer, cardiovascular disease, cerebrovascular disease, and also Diabetes, Liver disease which are the some of the main causes of death in Japan. Stiff neck is also regarded as the inevitable condition, if we consider the structure of the neck of human. The neck supports the head which is about $5 \mathrm{~kg}$ and also keeps flexibility at the same time with the seventh vertebra whose diameter is less than $3 \mathrm{~cm}$, and many other muscles or ligaments like the trapezius muscle, the nuchal muscle, the scalenus muscle, the sternocleidomastoid muscle. The following elements make the muscle strained, stressed, loose, and bring about the accumulation of fatigue, and hence the collapse of electrolyte balance and cause stiff neck; intense movement, continual fixed posture, repetition of the same operation, bad posture such as sloping shoulders or round shoulders.

The extreme stress of the motor nerve is also regarded as a cause of stiff neck. As such causes, the following are considered. neck discopathy, ${ }^{5}$ cervical deformans, cervical instability, thoracic outlet syndrome, periarthritis scapulohumeralis. The stimulus of the autonomous nerve also seems to cause the symptom of stiff neck.

It's also pointed out the relationship between an irregular teeth set and stiff neck. Once the teeth set becomes irregular, the sternocleidomastoid muscle of the same side becomes strained. Usually, the trapezius muscle, the scapular muscle and the rhomboids are regarded as the muscles, which cause the symptom of stiff neck, and the sternocleidomastoid muscle has the same core level or the same root as these muscles. So once the sternocleidomastoid muscle is strained due to irregular teeth set, it causes stiff neck ${ }^{6}$.

The disorders of neck, shoulder, and arms whose main symptoms are the stiffness of the affected parts, are considered to be caused by unbalanced body activities arising from the rapid development of the laborsaving environments and the change in life styles. ${ }^{7)}$ Where chronic stiff shoulder symptoms exist, it is also possible to consider the following as related factors: difficulty to adapt to society or workplace, undeveloped mentality, stress on the 
job. It also might be caused by inherent a hysteric and depressive condition appearing as a psychosomatic disease. ${ }^{81}$

In order to maintain a healthy condition it is also necessary to adapt to the present life and work environment by finding the balance between mental and physical aspects. ${ }^{9)}$ To prevent stiff neck the following conditions are in important: 1. keeping the correct posture, 2. do proper exercise. 3. keep a positive and active attitude. Continued research into the prevention of stiff neck will also be necessary.

\section{CONCLUSION}

As a result of this research, we could observe that there were a greater number of females $(50.0 \%)$ who suffer from stiff neck as opposed to the number of males $(28.5 \%)$. In many cases, the people who had stiff neck had the tendency to suffer from lumbago at the same time, feel stress, are less active in daily life, do less exercise, do not drink nor smoke. We would like to make use of these results of this research positively for the guidance to improve the living habits and life styles.

\section{REFERENCE}

[1] Motegi M., Etiology of Stiff neck, J Jap Orthop Surgery 1964; 38: 648.

[2] Japanese Ministry of Health and Welfare. Trend of National Hygiene (KOKUMIN EISEI NO DOUKOU) 1989, 1992, 1995, 2000.

[3] Yanaihara M., Actual condition of stiff neck by health examination data. J Chubu Orthop Hazard 1983; 26: 1166-1168.

[4] Charlton W.P., Coslett-Charlton L.M., Ciccotti M.G., Correlation of estradiol in pregnancy and anterior cruciate ligament laxity. Clin Orthop 2001 Jun; (387): 165-170.

[5] Hirabayashi K., Etiology and treatment of stiff neck. Clinics and Research (RINSHO TO KENKYU) 1993; 70-71: 199-204.

[6] Shinodaki K., Massage effect for head-neck syndrome derived from masseteric muscle. Physical Therapy (RIGAKURYOUHOUSHI), 1995; 23-3, 96-101.

[7] Osanai H., Health examination of neck, shoulder, arms syndrome (KEI-KEN-WAN-SHOKOGUN). Occupational Science (ROUDONO-KAGAKU). 1972; 27(8): 32-37.

[8] Matsumoto T., Experience in treatment of intractable head-neckshoulder syndrome. Occupational Science (ROUDONO-KAGAKU), 1977; 32(7) 18-23.

[9] Sasaki N., "Day of Bone and Joint" Press briefing, J Jap Clin Orthop. 2000; 25(2):1-2. 